\title{
Probióticos no Controle da Pré-Diabetes e Diabetes Tipo 2
}

\section{Probiotics in Management of Prediabetes and Diabetes Type 2 Control}

\author{
Yasmin Carla de Barros ${ }^{\mathrm{a}}$; Katia Sivieri*ab; Giselle Aparecida Nobre Costa ${ }^{\mathrm{a}}$ \\ ${ }^{a}$ Unopar, Programa de Pós-Graduação Stricto Sensu em Ciência e Tecnologia de Leite e Derivados. PR, Brasil.

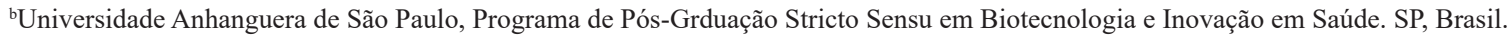 \\ *E-mail: katia.sivieri@kroton.com.br
}

\begin{abstract}
Resumo
Segundo a Organização Mundial da Saúde (OMS), o Diabetes Mellitus (DM), é uma doença grave e crônica, que ocorre quando o pâncreas não produz insulina suficiente, ou quando o corpo não é capaz de utilizar efetivamente a insulina produzida. A pré-diabetes pode ser observada em uma classe de indivíduos nos quais há um aumento dos níveis de glicose no sangue acima do normal e abaixo do diagnóstico de DM. Dados da Federação Internacional do Diabetes (IDF) e da Associação Americana de Diabetes (ADA) têm apontado para um grande aumento da prevalência da pré-diabetes e consequentemente de DM no mundo. Esse aumento da prevalência do diabetes está associado a diversos fatores, principalmente a fatores nutricionais, sedentarismo e a obesidade/sobrepeso. Esses distúrbios metabólicos são caracterizados por resistência à insulina e deficiência da mesma. Estudos indicam que a microbiota intestinal desempenha um importante papel na fisiopatologia da obesidade e consequentemente da pré-diabetes através de seus efeitos sobre o controle do peso corporal, balanço energético e inflamação. Neste contexto, a ingestão de probióticos vem mostrando efeitos positivos na modulação da microbiota intestinal e consequente controle da pré-diabetes e diabetes.
\end{abstract}

Palavras-chave: Diabetes. Pré-Diabetes. Obesidade. Microbiota Intestinal.

\begin{abstract}
According to the World Health Organization (WHO), Diabetes Mellitus (DM) is a serious and chronic disease, which occurs when the pancreas does not produce enough insulin, or when the body is not able to effectively use the insulin produced. Prediabetes can be seen in a class of individuals in whom there is an increase in blood glucose levels above normal and below the DM diagnosis. Data from the International Diabetes Federation (IDF) and the American Diabetes Association (ADA) have pointed to a great increase in the prevalence of pre-diabetes and consequently of DM in the world. This increase in the diabetes prevalence is associated with several factors, mainly nutritional factors, physical inactivity and obesity / overweight. These metabolic disorders are characterized by insulin resistance and insulin deficiency. Studies indicate that the intestinal microbiota plays an important role in the obesity pathophysiology and consequently prediabetes through its effects on body weight control, energy balance and inflammation. In this context, the intake of probiotics has been showing positive effects in the intestinal microbiota modulation and consequent pre-diabetes and diabetes control.
\end{abstract}

Keywords: Diabetes. Prediabetes. Obesity. Intestinal Microbiota.

\section{Introdução}

O Diabetes Mellitus - DM, é uma doença que ocorre quando o pâncreas não produz insulina suficiente, ou quando o corpo não é capaz de utilizar efetivamente a insulina produzida (OMS, 2016). A prevalência do diabetes está associada a diversos fatores, principalmente a fatores nutricionais, sedentarismo e a obesidade / sobrepeso (SBD, 2017).

Os principais tipos de DM são DM tipo 1, DM gestacional e DM tipo 2. Há ainda um grupo de indivíduos no qual são observados níveis de glicose no sangue acima do normal e abaixo do diagnóstico de DM, estes são classificado em pacientes com glicemia em jejum alterada (pré-diabéticos). Glicemia em jejum alterada (GJA) e tolerância à glicose diminuída - TGD são estágio intermediário na história natural do DM (ADA, 2014).

Estudos epidemiológicos revelam a relação entre obesidade e pré-diabetes. Ambos os distúrbios metabólicos são caracterizados por resistência à insulina e deficiência da mesma (LOIS, KUMAR, 2009; FELBER, GOLAY, 2002). Uma estimativa realizada por Hossain et al. (2007) estima que cerca de $90 \%$ do DM tipo 2 é atribuível ao excesso de peso.

Sabe-se que a microbiota intestinal desempenha importante papel na fisiopatologia da obesidade e consequentemente na pré-diabetes e diabetes por meio de seus efeitos sobre o controle do peso corporal, balanço energético e inflamação. Alguns estudos mostram diferenças na composição da microbiota intestinal e no metabolismo energético entre populações caracterizadas como magros ou obesos (LEY et al., 2006; TURNBAUGH et al., 2009) e que o consumo de alguns tipos de probióticos, tem efeitos benéficos na síndrome de resistência à insulina e na prevenção da obesidade (SÁEZLARA et al., 2016).

Diante do exposto, este estudo aborda como o consumo de probióticos podem influenciar positivamente o metabolismo da pré-diabetes e na diabetes. 


\section{Desenvolvimento}

\subsection{Metodologia}

Trata-se de uma revisão bibliográfica pautada em artigos científicos, bem como mídias impressas, tais como: jornais, revistas e periódicos.

Os artigos, teses, dissertações e monografias foram obtidos em bibliotecas digitais de Universidades e sites de pesquisa, como: SciELO, PubMed, Science Direct e Google Acadêmico. No campo de busca foi inserido o termo "diabetes", "prédiabetes", “obesidade", "sobrepeso", "probiótico", “ensaio clínico" e "microbiota intestinal".

\subsection{Discussão}

\subsubsection{Pré-diabetes e diabetes tipo 2}

Em 1997 e 2003, o Comitê de Especialistas em Diagnóstico e Classificação de Diabetes Mellitus reconheceu um grupo intermediário de indivíduos que não atendiam aos critérios para o diagnóstico de diabetes. Este grupo tinha como característica um nível de glicose maior do que o considerado normal. Tal grupo foi classificado em pacientes com glicemia em jejum alterada. Glicemia em jejum alterada - GJA e tolerância à glicose diminuída - TGD são estágios intermediários na história natural do DM (ADA, 2014). Distúrbios no metabolismo da glicose, GJA e TGD, caracterizam uma classe de indivíduos chamados de "prédiabéticos" e refletem a história natural da progressão da normoglicemia para o DM tipo 2 (IDF, 2017).

Em 2017 estimava-se que aproximadamente 300 milhões de indivíduos entre 20 e 79 anos apresentavam características que indicam risco futuro de desenvolver DM tipo 2. Em 2045, o número de pessoas de 20 a 79 anos com TGD está projetado para aumentar para cerca de 600 milhões (IDF, 2017).

Já a DM tipo 2 é um distúrbio metabólico caracterizado por alta glicose no sangue resultante de uma combinação de secreção insuficiente de insulina e resistência à insulina (ASEMI et al. 2013). Embora o DM tipo 2 seja mais comumente diagnosticado em idosos, a incidência dessa patologia tem sido cada vez mais observada em crianças, adolescentes e adultos jovens devido ao aumento níveis de obesidade, inatividade física e dieta pobre (IDF 2017).

Os fatores e mecanismos que desencadeiam a pré-diabetes e a DM tipo 2 são: idade avançada, dieta desequilibrada, sedentarismo, tabagismo, histórico familiar e em especial a obesidade e o sobrepeso (VAZQUEZ; DUVAL; JACOBS, 2007; FOROUZANFAR, 2015).

\subsubsection{Microbiota intestinal e obesidade}

A OMS define sobrepeso e obesidade como acúmulo anormal ou excessivo de gordura que apresenta risco à saúde. A medida utilizada para determinar o grau de sobrepeso ou obesidade de uma pessoa é o índice de massa corporal IMC. Uma pessoa com um IMC de 30 ou mais é geralmente considerada obesa (OMS, 2019).

Estudos epidemiológicos revelam a relação entre obesidade e diabetes. Ambos os distúrbios metabólicos são caracterizados por resistência à insulina e deficiência da mesma (LOIS, KUMAR, 2009; FELBER, GOLAY, 2002). Uma estimativa realizada por Hossain et al. (2007) estima que cerca de $90 \%$ do DM tipo 2 é associada ao excesso de peso.

A causa da obesidade não pode ser definida apenas por um desequilíbrio energético entre as calorias consumidas e as calorias gastas, mas também por influência de fatores genéticos, fatores ambientais, comportamentais, psicológicos, fisiológicos e/ou alteração da microbiota intestinal (LEY et al., 2006; SANZ; SANTACRUZ; GAUFFIN, 2010; ZHANG et al., 2009).

Um adulto saudável abriga 500-1000 espécies bacterianas, cerca de $10^{12}-10^{14}$ microrganismos em todo o intestino com uma massa de cerca de 1-2 kg (BLAUT; CLAVEL, 2017). Abordagens metagenômicas revelaram que aproximadamente $90 \%$ das espécies bacterianas presentes no intestino de adultos pertencem aos filos Bacteroidetes e Firmicutes (Grampositivos) (HUMAN ${ }^{\mathrm{a}}, 2015$; HUMAN $^{\mathrm{b}}, 2015$ ).

A microbiota intestinal tem sido associada à fisiopatologia da obesidade e consequentemente do pré-diabetes e na diabetes através de seus efeitos sobre o controle do peso corporal, balanço energético e inflamação. Alguns estudos mostram diferenças na composição da microbiota intestinal e no metabolismo energético entre populações que são magros ou obesos (LEY et al., 2006; TURNBAUGH et al., 2009).

Estudos na caracterização da microbiota intestinal de indivíduos com DM tipo 2 e na avaliação de possível correlações entre abundância de certos grupos e aspectos metabólicos têm relatado que pacientes com DM tipo 2 apresentam disbiose, e consequentemente um alto número de bactérias patogênicas oportunistas, tais como; Clostridium clostridioforme, Bacteroides caccae, Clostridium hathewayi, Clostridium ramosum, Clostridium symbiosum, Eggerthella sp., e Escherichia coli (KARLSSON et al., 2013; LARSEN et al., 2010).

De acordo com Sabatino et al., (2017) as principais características na microbiota de pacientes com DM tipo 2 são: redução de bactérias produtoras de (Roseburia intestinalis e Faecalibacterium prausnitzii), disbiose moderada, ambiente pró-inflamatório com aumento da expressão de genes microbianos envolvidos no estresse oxidativo, expressão reduzida de genes envolvidos na síntese de vitaminas, concentração sérica aumentada de LPS e aumento da permeabilidade intestinal.

Apesar das diferenças na composição da microbiota intestinal de obesos e magros ainda serem questionáveis, predições do metabolismo da comunidade microbiana, baseadas em conteúdo dos genes, indicam que a microbiota associada a obesos possui uma capacidade maior em coletar energia dos alimentos, por meio da quebra mais eficiente de 
polissacarídeos (THE HUMAN MICROBIOME PROJECT CONSORTIUM, 2012). Turnbaugh et al. (2006) observaram menor quantidade de energia remanescente em fezes de camundongos obesos em relação a seus homólogos magros, evidenciando a capacidade aumentada que o microbioma obeso possui em extrair energia da dieta.

Curiosamente, o fenótipo de coleta de energia também pode ser transmissível simplesmente pelo transplante da "microbiota obesa". A colonização de camundongos isentos de bactérias (germ-free) com uma 'microbiota obesa' resultou em um aumento significativo na gordura corporal quando comparado com os animais colonizados por uma 'microbiota magra' (TURNBAUGH et al., 2006, 2009).

\subsubsection{Probióticos e a microbiota intestinal no controle da pré-diabetes e diabetes}

A primeira observação do papel positivo no organismo humano, desempenhado por algumas bactérias selecionadas, é atribuída ao russo Eli Metchnikoff, ganhador do Prêmio Nobel de medicina quando trabalhava no Instituto Pasteur, no início do século passado. Sua teoria dizia que a saúde poderia ser melhorada, e também que a senilidade poderia ser retardada, manipulando o microbioma intestinal através de bactérias benéficas para o hospedeiro encontradas no iogurte (METCHNIKOFF, 1910).

Os probióticos são microrganismos vivos que, quando administrados em quantidades adequadas, conferem benefícios à saúde do hospedeiro (HILL et al., 2014).

Membros dos gêneros Lactobacillus e Bifidobacterium são os mais utilizados como microrganismos probióticos (FAO, 2006).
Os possíveis mecanismos de ação dos probióticos são a produção de compostos com atividade antimicrobiana, competição por nutrientes ou por sítios de adesão (aumento da função de barreira do epitélio), diminuição da permeabilidade intestinal e modulação do sistema imune (FULLER, 1991; SETH et al., 2008; ANDERSON et al., 2010; KARCZEWSKI et al., 2010; SAARELA et al., 2000; BRON et al., 2012).

Sabe-se que uma alimentação saudável com inclusão de probióticos e produtos lácteos fermentados, são indicados para aumentar a diversidade da microbiota intestinal, a fim de obter-se um impacto benéfico sobre a obesidade através da restauração de uma microbiota saudável e seu papel regulador no eixo intestino-cérebro (MARETTE, PICARD-DELAND, 2014; BUHMANN et al., 2014; GRAF et al., 2015;).

Há ainda evidências de que o consumo de produtos lácteos contendo probióticos resulta na redução do colesterol sanguíneo, que pode ser útil na prevenção da obesidade, diabetes, doenças cardiovasculares e acidente vascular cerebral (AVC) (SIMONS; AMANSEC; CONWAY, 2006).

Nos Quadros 1 e 2 são apontados os principais estudos e efeitos da ingestão de probióticos em pacientes pré-diabéticos (Quadro 1) ou diabéticos (Quadro 2). Na maioria destes ensaios clínicos foram utilizadas cepas de Lactobacillus ou Bifidobacterium. O tempo de intervenção variou de 4 semanas a 4 meses. A dose diária foi acima de $10^{9}$ UFC. Os resultados encontrados após a ingestão dos probióticos foram promissores, tais como a redução na hiperglicemia (KASSAIN et al., 2018), diminuição da glicose em jejum (RAZMPOOSH et al., 2018) e redução da resistência à insulina (KARAMALI et al., 2016; KOBYLIAK et al. 2018).

Quadro 1 - Efeito das diferentes espécies de probióticos na pré-diabetes (ensaio clínico)

\begin{tabular}{|c|c|c|c|c|}
\hline Probiótico & Grupo & Dose/Duração & Principais Achados & Ref. \\
\hline $\begin{array}{l}\text { Lactobacillus } \\
\text { acidophilus, } \\
\text { Bifidobacterium bifidum, } \\
\text { Bifidobacterium lactis, e } \\
\text { Bifidobacterium longum }\end{array}$ & $\begin{array}{l}85 \text { adultos com pré-diabetes } \\
\text { concluíram o estudo. Os } \\
\text { indivíduos foram divididos } \\
\text { aleatoriamente em } 3 \text { grupos: } \\
\mathrm{N}=27 \text { no grupo probiótico, } \\
30 \text { no grupo simbiótico e } \\
\mathrm{N}=28 \text { no grupo placebo. }\end{array}$ & $\begin{array}{c}\text { Grupo probiótico: } 6 \mathrm{~g} / \mathrm{d} \\
\text { cultura probiótica }\left(1.5 \text { x } 10^{9}\right. \\
\text { UFC) } \\
\text { Grupo simbiótico: } \\
\text { probióticos + inulina } \\
\text { Grupo placebo: } \\
\text { maltodextrina. } \\
\text { Duração: } 24 \text { semanas. }\end{array}$ & $\begin{array}{l}\text { - Redução na hiperglicemia } \\
\text { nos gupos probiótico e } \\
\text { simbiótico e da hipertensão no } \\
\text { grupo probiótico. } \\
\text { - Redução na prevalência } \\
\text { da síndrome metabólica } \\
\text { foi significativa após a } \\
\text { suplementação probiótica e } \\
\text { simbiótica em comparação } \\
\text { com o placebo. } \\
\text { - Diminuição do HDL no } \\
\text { grupo probiótico. }\end{array}$ & $\begin{array}{l}\text { KASSAIAN, } \\
\mathrm{N} . \text { et al., } \\
2018 .\end{array}$ \\
\hline $\begin{array}{l}\text { Lactobacillus casei } \\
\text { Shirota (LcS) }\end{array}$ & $\begin{array}{l}100 \text { japoneses pré- } \\
\text { diabéticos obesos } \\
\text { Os indivíduos foram } \\
\text { divididos aleatoriamente } \\
\text { em } 2 \text { grupos: } \mathrm{N}=50 \text { grupo } \\
\text { probiótico (LcS), N=50 } \\
\text { grupo placebo. }\end{array}$ & $\begin{array}{c}\text { Grupo probiótico: } 100 \\
\text { ml de leite fermentado } \\
\text { contendo LcS }(>1.0 \times \\
1011 \text { UFC) consumida } \\
\text { diariamente. } \\
\text { Grupo placebo: } 100 \mathrm{ml} \\
\text { de placebo foi consumida } \\
\text { diariamente. } \\
\text { Duração: } 8 \text { semanas. }\end{array}$ & $\begin{array}{l}\text { - Os níveis de hiperglicemia } \\
\text { pós-carga (PG) após } 1 \text { hora de } \\
\text { carga, glicoalbumina e HbA1c } \\
\text { diminuíram no grupo LcS. } \\
\text { - A redução nos níveis de } \\
\text { glicoalbumina foi maior no } \\
\text { grupo LcS do que no grupo } \\
\text { placebo } \\
\text {-Níveis totais de lipoproteína } \\
\text { de baixa densidade e de } \\
\text { lipoproteína de alta densidade } \\
\text { foram menores no grupo LcS } \\
\text { do que no grupo placebo }\end{array}$ & $\begin{array}{l}\text { NAITO, E. et } \\
\text { al., } 2018\end{array}$ \\
\hline
\end{tabular}


Streptococcus

thermophilus,

Bifidobacteria breve,

Bifidobacteria longum,

Bifidobacteria infantis,

Lactobacillus

acidophilus,

Lactobacillus

plantarum,

Lactobacillus paracasei,

Lactobacillus

delbreuckii

subspecies bulgaricus

Fonte: Dados da pesquisa.
Grupo probiótico:

Administração de sachês com probióticos contendo $450 \times 10^{9}$ UFC, duas vezes ao dia e aconselhamento para um estilo de vida mais - Pequenas diferenças foram saudável.

Grupo placebo:

Aconselhamento para um estilo de vida mais saudável.

Duração: 4 meses. reveladas no status de controle glicêmico e homeostase

STEFANAKI et al., 2019.

Quadro 2 - Efeito das diferentes espécies de probióticos na diabetes (ensaio clínico)

\begin{tabular}{|c|c|c|c|c|}
\hline Probiótico & Grupo & Dose/Duração & Principais achados & Ref. \\
\hline $\begin{array}{l}\text { Lactobacillus } \\
\text { acidophilus, } \\
\text { Lactobacillus casei, } \\
\text { Lactobacillus } \\
\text { rhamnosus, } \\
\text { Lactobacillus } \\
\text { bulgaricus, } \\
\text { Bifidobacterium breve, } \\
\text { Bifidobacterium } \\
\text { longum, Streptococcus } \\
\text { thermophilus }\end{array}$ & $\begin{array}{l}60 \text { pacientes com DM tipo } 2 \\
\text { foram recrutados para este } \\
\text { estudo. } \\
\text { Os indivíduos foram } \\
\text { divididos aleatoriamente } \\
\text { em } 2 \text { grupos: } \mathrm{N}=30 \text { grupo } \\
\text { probiótico (LcS), N=30 } \\
\text { grupo placebo }\end{array}$ & $\begin{array}{c}\text { Grupo probiótico: } \\
\text { administração } \\
\text { de cápsula contendo } \\
\text { suplemento probiótico. } \\
\text { Grupo placebo: } \\
\text { administração } \\
\text { de cápsula contendo } \\
\text { placebo. } \\
\text { Duração: } 6 \text { semanas. }\end{array}$ & $\begin{array}{l}\text { - Diminuição e aumento } \\
\text { significativos nos níveis } \\
\text { de glicose em jejum (GJ) e } \\
\text { HDL-C no grupo probiótico, } \\
\text { respectivamente. }\end{array}$ & $\begin{array}{l}\text { RAZMPOOSH, } \\
\text { E. et al., } 2018 .\end{array}$ \\
\hline $\begin{array}{l}\text { Mistura probiótica } \\
\text { contendo biomassa de } \\
14 \text { cepas: Lactobacillus, } \\
\text { L a c to co c c u s, } \\
\text { B ifidobacterium, } \\
\text { Propionibacterium, } \\
\text { Acetobacter. }\end{array}$ & $\begin{array}{l}53 \text { pacientes com DM tipo } 2 \\
\text { Os indivíduos foram } \\
\text { divididos aleatoriamente } \\
\text { em } 2 \text { grupos: } \mathrm{N}=30 \text { grupo } \\
\text { probiótico (LcS), N=30 } \\
\text { grupo placebo }\end{array}$ & $\begin{array}{c}\text { Grupo probiótico: } \\
\text { administração de } \\
\text { multiprobiótico (biomassa } \\
\text { concentrada com } 14 \\
\text { gêneros de bactérias } \\
\text { probióticas) na forma de } \\
\text { sachê. } \\
\text { Grupo placebo: } \\
\text { administração de placebo } \\
\text { como uma formulação de } \\
\text { sachê. } \\
\text { Duração: } 8 \text { semanas. }\end{array}$ & $\begin{array}{l}\text {-Redução da resistência } \\
\text { à insulina no grupo } \\
\text { probiótico. }\end{array}$ & $\begin{array}{l}\text { KOBYLIAK, N., } \\
\text { et al. } 2018\end{array}$ \\
\hline 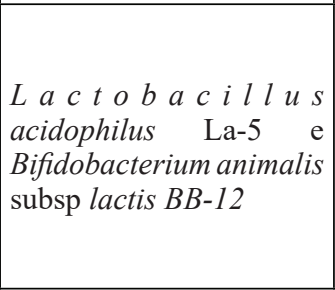 & $\begin{array}{l}50 \text { pacientes com DM tipo } \\
2 . \quad \text { indivíduos foram } \\
\text { Os aleatoriamente } \\
\text { divididos grupos: } \mathrm{N}=25 \text { grupo } \\
\text { em } 2 \text { grupo } \\
\text { probiótico, } \mathrm{N}=25 \text { grupo } \\
\text { placebo }\end{array}$ & $\begin{array}{c}\text { Grupo probiótico: } \\
\text { administração de } 120 \mathrm{~g} \\
\text { / d de leite fermentado } \\
10^{9}(\text { UFC). } \\
\text { Grupo placebo: } \\
\text { administração de leite } \\
\text { fermentado convencional } \\
\text { Duração: } 6 \text { semanas. }\end{array}$ & $\begin{array}{l}\text { Redução níveis de } \\
\text { frutosamina e hemoglobina } \\
\text { Alc no grupo probiótico. } \\
\text { - Houve uma diferença } \\
\text { significativa entre os grupos } \\
\text { quanto às alterações médias } \\
\text { da HbAlc, colesterol total } \\
\text { e colesterol LDL. }\end{array}$ & $\begin{array}{l}\text { TONUCCI, L. et } \\
\text { al., } 2015 .\end{array}$ \\
\hline 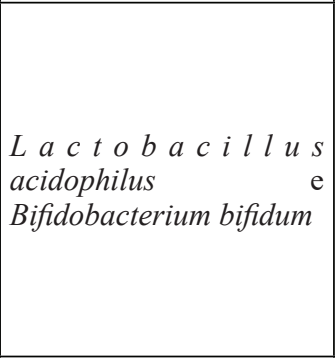 & $\begin{array}{l}57 \text { mulheres grávidas com } \\
\text { DM gestacional (DMG) } \\
\text { com } 24 \text { a } 28 \text { semanas de } \\
\text { gestação. } \\
\begin{array}{l}\text { Divididas aleatoriamente } \\
\text { em } 2 \text { grupos: } \mathrm{N}=28 \text { grupo } \\
\text { probiótico, N=29 grupo } \\
\text { placebo }\end{array}\end{array}$ & $\begin{array}{c}\text { Grupo probiótico: } \\
\text { administração de capsula } \\
\text { contendo suplementos } \\
\text { probióticos }\left(10^{9} \text { UFC }\right) \\
\text { diariamente. } \\
\text { Grupo placebo: } \\
\text { administração capsula } \\
\text { contendo placebo } \\
\text { diariamente } \\
\text { Duração: } 4 \text { semanas. }\end{array}$ & $\begin{array}{l}\text { - Melhora no metabolismo } \\
\text { da glicose no grupo } \\
\text { probiótico em comparação } \\
\text { ao grupo placebo. }\end{array}$ & $\begin{array}{l}\text { KIJMANAWAT, } \\
\text { A., et al., } 2018 .\end{array}$ \\
\hline 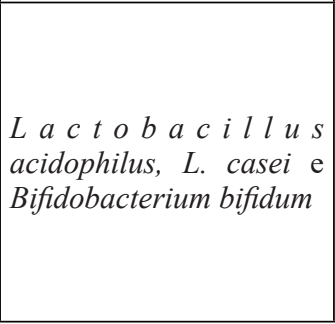 & $\begin{array}{l}\text { Sessenta mulheres grávidas } \\
\text { com DMG, primigestas e } \\
\text { com idades entre } 18 \text { e } 40 \\
\text { anos. } \\
\text { Divididos aleatoriamente } \\
\text { em } 2 \text { grupos: } \mathrm{N}=30 \text { grupo } \\
\text { probiótico, } \mathrm{N}=30 \text { grupo } \\
\text { placebo. }\end{array}$ & $\begin{array}{c}\text { Grupo probiótico: } \\
\text { administração de capsula } \\
\text { contendo suplementos } \\
\text { probióticos }\left(2 \times 10^{9} \text { UFC). }\right. \\
\text { Grupo placebo: } \\
\text { administração capsula } \\
\text { contendo placebo } \\
\text { (celulose). } \\
\text { Duração: } 6 \text { semanas. }\end{array}$ & $\begin{array}{l}\text { - Reduções significativas } \\
\text { na GJ } \\
\text { - Aumento significativo } \\
\text { no índice quantitativo de } \\
\text { sensibilidade à insulina }\end{array}$ & $\begin{array}{l}\text { K A R A M A L I, } \\
\text { M., et al., } 2016 .\end{array}$ \\
\hline
\end{tabular}




\begin{tabular}{|c|c|c|c|c|}
\hline $\begin{array}{l}\text { Bifidobacterium bifidum, } \\
\text { B ifid o b a c te ri } \text { i m } \\
\text { lactis, Lactobacillus } \\
\text { a c id o p h i l u s, } \\
\text { Lactobacillus brevis, } \\
\text { Lactobacillus casei, } \\
\text { Lactobacillus salivarius, } \\
\text { Lactococcus lactis e L. } \\
\text { lactis }\end{array}$ & $\begin{array}{l}30 \text { indivíduos com DM tipo } \\
2 \text { foram recrutados para este } \\
\text { estudo. }\end{array}$ & $\begin{array}{c}\text { Grupo probiótico: } \\
\text { administração de cápsula } \\
\text { contendo suplementos } \\
\text { probióticos }\left(2,5 \times 10^{9} \text { UFC }\right) \\
\text { diariamente. } \\
\text { Grupo placebo: } \\
\text { administração cápsula } \\
\text { contendo placebo } \\
\text { diariamente } \\
\text { Duração: } 6 \text { meses. }\end{array}$ & $\begin{array}{l}\text {-Diminuição do indicador } \\
\text { RI em pacientes com DM } \\
\text { tipo2. }\end{array}$ & $\begin{array}{l}\text { SABICO, S., et } \\
\text { al., } 2018 .\end{array}$ \\
\hline
\end{tabular}

Fonte: Dados da pesquisa.

\subsubsection{Probióticos no metabolismo de ácidos graxos de cadeia curta (AGCC) e a sua influência no controle da pré- diabetes e diabetes}

Os AGCC são provenientes da fermentação dos hidratos de carbono não digeríveis, incluindo a celulose, xilanas, amido resistente e inulina, no cólon pelas bactérias anaeróbicas (TREMAROLI; BACKHED, 2012). Os AGCCs (acetato, propionato e butirato) produzido pela microbiota intestinal desempenham papéis importantes como substratos para o metabolismo de glicose, colesterol e lipídios (DEN BESTEN et al., 2013).

Evidências científicas demonstram que alguns tipos de probióticos, como por exemplo Bifidobacterium, tem efeitos benéficos na síndrome de resistência à insulina, no metabolismo de ácidos graxos de cadeia curta (AGCC) e sobretudo na diversidade da microbiota (SÁEZ-LARA et al., 2016)

Um dos efeitos na saúde atribuídos à produção de AGCC é a redução do $\mathrm{pH}$ luminal, que inibe microrganismos patogênicos e aumenta a absorção de alguns nutrientes (MACFARLANE et al, 2012). O acetato é um elemento chave na capacidade de as bifidobactérias inibirem os enteropatógenos (FUKUDA et al., 2011).

O envolvimento de AGCC no metabolismo energético e lipídico atraiu a atenção dos pesquisadores para o papel potencial destes no controle da síndrome metabólica. Uma redução na obesidade e resistência à insulina em animais experimentais em dieta rica em gordura após a suplementação dietética com butirato foi observada (GAO et al., 2009).

\subsubsection{Nova geração de probióticos}

O avanço das técnicas moleculares, ou seja, independentes de cultivo, na avaliação da microbiota intestinal vem revelando a importância de bactérias intestinais comensais no desenvolvimento da saúde do hospedeiro, as quais estão intimamente associadas à progressão da gravidade de doenças específicas (CHANG et al., 2019). Assim, algumas cepas bacterianas intestinais comensais não caracterizadas anteriormente são atualmente referidas como Nova Geração de Probióticos - NGP. NGP são espécies e cepas bacterianas pertencentes aos filos Actinobacteria, Bacteroidetes, Firmicutes e Verrucomicrobia (LIN, et al., 2019).

Interessantemente, alguns estudos recentes revelaram muitos NGP como ferramenta no controle da pré-diabetes e diabetes (CHANG et al., 2019). Estes incluem Prevotella copri e Christensenella minuta para o controle da resistência à insulina (LI et al., 2010), Parabacteroides goldsteinii, Akkermansia muciniphila, Bacteroides thetaiotaomicron na reversão da obesidade e resistência à insulina (CANI et al., 2018).

Entretanto, os NGP selecionados em modelo in vitro devem ser rigorosamente avaliados conforme os protocolos de segurança propostos pelos órgãos regulamentadores (HILL et al., 2014). Atualmente, o FDA nos Estados Unidos iniciou um programa de produtos bio-terapêuticos vivos para regular especificamente a aplicação, ensaio clínico e comercialização de NGP (CHANG et al., 2019). Espera-se que, em um futuro próximo, que novos probióticos comecem a se tornar realidade na indústria da biofármacos.

\section{Conclusão}

A pré-diabetes e a diabetes são doenças crônicas que afetam um grande número de indivíduos em todo o mundo, cada vez mais, a microbiota intestinal tem sido associada a essas patologias. Esta revisão evidencia a importância da composição da microbiota e os mecanismos envolvidos nestas patologias. O consumo de probióticos, principalmente, Lactobacillus spp., Bifidobacterium spp, é uma promissora estratégia no controle da pré-diabetes e da diabetes. O consumo de probióticos, também, influência positivamente no metabolismo de AGCC, os quais desempenham papéis importantes na saúde do hospedeiro.

Há ainda um vasto campo de investigação sobre os probióticos e seus modos de ação em doenças crônicas, assim como a introdução de novas espécies e linhagens que possam contribuir na prevenção das doenças do século XXI, tais como a pré-diabetes e diabetes.

\section{Agradecimentos}

A CAPES, pela taxa concedida para a primeira autora. Ao Programa de Pós-Graduação em Ciência e Tecnologia de Leite e Derivados, da UNOPAR.

\section{Referências}

ADA - American Diabetes Association. Diagnosis and classification of diabetes mellitus. Diabetes Care, v.37, p.581590, 2014.

ANDERSON, R.C. et al. Lactobacillus plantarum MB452 
enhances the function of the intestinal barrier by increasing the expression levels of genes involved in tight junction formation. BMC Microbiology, v.10, p.316, 2010.

ASEMI, Z. et al. Effect of multispecies probiotic supplements on metabolic profiles, hs-CRP, and oxidative stress in patients with type 2 diabetes. Ann. Nutr. Metab., v.63, n.1/2, p.1-9, 2013.

BLAUT M., CLAVEL T. Metabolic diversity of the intestinal microbiota: implications for health and disease. J. Nutr., v.137, p.751-755, 2017. doi: 10.1093/jn/137.3.751S.

BRON, P.A.; VAN BAARLEN, P.; KLEEREBEZEM, M. Emerging molecular insights into the interaction between probiotics and the host intestinal mucosa. Nat. Rev. Microbiol., v.10, p.66-U90, 2012.

BUHMANN, H.; LE ROUX ,C.W.; BUETER, M. The gut-brain axis in obesity. Best Practice \& Research. Clin. Gastroenterol., v.28, n.4, p.559-571, 2014.

CANI, P.D. Human gut microbiome: hopes, threats and promises. Gut, v.67, p.1716-1725, 2018. doi: http://dx.doi.org/10.1136/ gutjnl-2018-316723

CHANG, C.J. et al. Next generation probiotics in disease amelioration. J. Food Drug Analysis, v.27, p.615-622, 2019. doi: https://doi.org/10.1016/j.jfda.2018.12.011

DEN BESTEN G. et al. The role of short-chain fatty acids in the interplay between diet, gut microbiota, and host energy metabolism. J. Lipid. Res., v.54, p.2325-2340, 2013.

FAO, Probiotics in Food: health and nutricional properties and guidelines for evaluation. Rome: Food and Agriculture Organization of the United Nations, 2006. Disponível em: http:// www.fao.org/3/a-a0512e.pdf

FELBER, J.P,; GOLAY, A. Pathways from obesity to diabetes. Int. J. Obes. Relat. Metab. Disord., v.26, n.2, p.39-45, 2002.

FOROUZANFAR, M.H. et al. Global,regional, and national comparative risk assessment of 79 behavioural,environmental and occupational,and metabolic risks or clusters of risks in 188 countries, 1990-2013: a systematic analysis for the Global Burden of Disease Study 2013. Lancet. v.386, p.2287-2323, 2015.

FUKUDA, S. et al. Bifidobacteria can protect from enteropathogenic infection through production of acetate. Nature, v.469, p.543-547, 2011.

FULLER, R. Probiotics in human medicine. Gut, v.32, p.439442, 1991.

GAO, Z. et al. Butyrate improves insulin sensitivity and increases energy expenditure in mice. Diabetes, v.58, p.1509-1517, 2009

GRAF, D. et al. Contribution of diet to the composition of the human gut microbiota. Microbial Ecol. Health Dis., v.26, n. 26164, 2015. doi: 10.3402/mehd.v26.26164.

HOSSAIN, P.; KAWAR, B.; EL NAHAS, M. Obesity and diabetes in the developing world-a growing challenge. N. Engl. J. Med., v.356, n.3, p.213-215, 2007.

HILL, C. et al. Expert consensus document. The International Scientific Association for Probiotics and Prebiotics consensus statement on the scope and appropriate use of the term probiotic. Nat. Rev. Gastroenterol. Hepatol., v.11, p.506-514, 2014.

HUMAN MICROBIOME PROJECT C. A framework for human microbiome research. Nature, v.486, p.215-221, 2015a.

HUMAN MICROBIOME PROJECT C. Structure, function and diversity of the healthy human microbiome. Nature, v.486, p.207$214,2015 b$

IDF- International Federation of Diabetes. Diabetes Atlas.
Bélgica: IDF, 2017.

INTERNATIONAL DIABETES FEDERATION (IDF). Diabetes Atlas. 2017. Disponível em: https://diabetesatlas.org

KARAMALI, M. et al. Effects of probiotic supplementation on glycaemic control and lipid profiles in gestational diabetes: a randomized, double-blind, placebo-controlled trial. Diabetes Metabolism., v.42, p.234-241, 2016. doi: 10.1016/j. diabet.2016.04.009

KARCZEWSKI, J. et al. Regulation of human epithelial tight junction proteins by Lactobacillus plantarum in vivo and protective effects on the epithelial barrier. Am. J. Physiol.Gastrointest Liver Physioliol., v.298, p.851-859, 2010

KARLSSON, F.H. et al. Gut metagenome in European women with normal, impaired and diabetic glucose control. Nature, v.498, p.99-103, 2013

KASSAIAN, N. et al. Probiotic and synbiotic supplementation could improve metabolic syndrome in prediabetic adults: a randomized controlled trial. Diabetes Metab. Syndrome Clin. Res. Rev., v.13, p.2991-2996, 2018. doi: 10.1016/j.dsx.2018.07.016

KIJMANAWAT, A. et al. Effects of probiotic supplements on insulin resistance in gestational diabetes mellitus: a double-blind randomized controlled trial. J. Diabetes Investig., v.10, p.163170, 2019. doi: 10.1111/jdi.12863

KOBYLIAK, N. et al. Effect of alive probiotic on insulin resistance in type 2 diabetes patients: Randomized clinical trial. Diabetes Metab. Syndrome Clin. Res. Rev., v.12, p.617-624, 2018. doi: https://doi.org/10.1016/j.dsx.2018.04.015

LARSEN, N. et al. Gut microbiota in human adults with type 2 diabetes differs from non-diabetic adults. PLOS ONE, v.5, p.e9085, 2010.

LEY, R.E. Microbial ecology: human gut microbes associated with obesity. Nature. v.444, n.7122, p.1022-1023, 2006.

LOIS, K.; KUMAR, S. Obesity and diabetes. Endocrinol. Nutr., v.56, n.4, p.38-42, 2009.

LIN, T.L. et al. Investiture of next generation probiotics on amelioration of diseases: strains do matter. Med. Microecol., v.1/2, p.1-7, 2019. doi: 10.1016/j.medmic.2019.100002

MACFARLANE, G.T.; MACFARLANE, S. Bacteria, colonic fermentation, and gastrointestinal health. J. AOAC Int., v. 95, p.50-60 2012 .

MARETTE, A.; PICARD-DELAND, E. Yogurt consumption and impact on health: Focus on children and cardiometabolic risk. Am. J. Clin. Nutr., v.99, p.1243S-1247S, 2014.

METCHNIKOFF, E. In: Mitchell PC. The prolongation of life. New York: Optimistic Studies, 1910.

NAITO, E., et al. Effect of Lactobacillus casei strain Shirotafermented milk on metabolic abnormalities in obese prediabetic Japanese men: a randomised, double-blind, placebo-controlled trial. Biosc. Microb. Food Health, v.37, p.9-18, 2018

OMS- World Health Organization. Global report on diabetes. Geneva: OMS, 2016.

OMS - World Health Organization. Obesity. Geneva: OMS, 2019.

RAZMPOOSH, E. et al. The effect of probiotic supplementation on glycemic control and lipid profile in patients with type 2 diabetes: a randomized placebo controlled trial. Diabetes Metabolic Syndrome Clin. Res. Rev., v.13, p.175-182, 2018.

SABATINO, A. et al. Intestinal microbiota in type 2 diabetes and chronic kidney disease. Current Diabetes Rep.s, v.3, p.16- 25, 


\section{10.1007/s11892-017-0841-z}

SAARELA, M. et al. Probiotic bacteria: safety, functional and technological properties. J. Biotechnol., v.84, p.197-215, 2000.

SABICO, S. et al. Effects of a 6-month multi-strain probiotics supplementation in endotoxemic, inflammatory and cardiometabolic status of $\mathrm{t} 2 \mathrm{dm}$ patients: a randomized, doubleblind, placebo-controlled trial. Clin. Nutr., v.38, p.1561-1569, 2018. doi: 10.1016/j.clnu.2018.08.009

SÁEZ-LARA, M.J. et al. Effects of probiotics and synbiotics on obesity, insulin resistance syndrome, type 2 diabetes and nonalcoholic fatty liver disease: a review of human clinical trials. Int. J. Mol. Sci., v.17, n.6, p.928, 2016. doi: 10.3390/ijms17060928

SANZ, Y.; SANTACRUZ, A.; GAUFFIN, P. Gut microbiota in obesity and metabolic disorders Proc. Nutr. Soc., v.69, n.3, p.434441, 2010.

SBD - Sociedade Brasileira de Diabetes. Diretrizes da Sociedade Brasileira de Diabetes 2017-2018. 2017. Disponível em: https:// www.diabetes.org.br/profissionais/images/2017/diretrizes/ diretrizes-sbd-2017-2018.pdf

SETH, A. et al. Probiotics ameliorate the hydrogen peroxideinduced epithelial barrier disruption by a PKC- and MAP kinasedependent mechanism. Am. J. Physiol. Gastroint. Liver Physiol., v.294, p.1060-1069, 2008.

SIMONS, L.A.; AMANSEC, S.G.; CONWAY, P. Effect of Lactobacillus fermentum on serum lipids in subjects with elevated serum cholesterol. Nutr. Metab. Cardiovasc. Dis., v.16, p.531-535, 2006.
STEFANAKI, C. et al. Probiotics in Adolescent Prediabetes: a pilot RCT on glycemic control and intestinal bacteriome. J. Clin. Med., v.8 p.1743, 2019. doi: 10.3390/jem8101743

THE HUMAN MICROBIOME PROJECT CONSORTIUM. Structure, function and diversity of the healthy human microbiome. Nature, v. 486, p. 207-214, 2012.

TONUCCI, L.B. et al. Clinical application of probiotics in type 2 diabetes mellitus: a randomized, double-blind, placebocontrolled study. Clin. Nutr., v.36, p.85-92, 2015. doi: 10.1016/j. clnu.2015.11.011.

TREMAROLI, V.; BACKHED, F. Functional interactions between the gut micro-biota and host metabolism. Nature, v.489, p.242-249, 2012

TURNBAUGH, P.J. et al. An obesity-associated gut microbiome with increased capacity for energy harvest. Nature, v.444, p.10271031, 2006

TURNBAUGH, P.J. et al. A core gut microbiome in obese and lean twins. Nature, v.457, n.7228, p.480-484, 2009.

VAZQUEZ, G.; DUVAL, S.; JACOBS, D.R. Comparison of body mass index, waist circumference, and waist/hip ratio in predicting incident diabetes: a meta-analysis. Epidemiol. Rev., v.29, p. 115128, 2007.

ZHANG $\mathrm{H}$. et al. Human gut microbiota in obesity and after gastric bypass. Pro.c Natl. Acad. Sci. USA. v.106, n.7, p.23652370, 2009. 